\title{
Deep learning of multibody minimal coordinates
}

\author{
Andrea Angeli ${ }^{1,2, *}$, Frank Naets ${ }^{1,2,}$, and Wim Desmet ${ }^{1,2}$, \\ ${ }^{1}$ LMSD, Department of Mechanical Engineering, KU Leuven \\ ${ }^{2}$ DMMS Lab, Flanders Make
}

Multibody systems [1] are the state-of-the-art tool to model complex mechanical mechanisms. However, they typically include redundant coordinates plus constraints, leading to differential algebraic equations for the dynamics which require dedicated integration schemes and control/estimation algorithms.

In the deep learning field, autoencoders are a particular neural network class with a symmetric structure to perform a nonlinear dimensionality reduction. In fact, they can be seen as a non-linear extension of principal component analysis [2].

In this work, it is proposed to use an autoencoder to reduce a general multibody model to minimal coordinates, eliminating the constraints. The obtained decoder function is used to perform a non-linear projection of the multibody coordinates:

$$
\begin{aligned}
\boldsymbol{x} & =\boldsymbol{h}_{e}(\boldsymbol{q}) \\
\tilde{\boldsymbol{q}} & =\boldsymbol{h}_{d}(\boldsymbol{x}) \\
\dot{\tilde{\boldsymbol{q}}} & =\frac{\partial \boldsymbol{h}_{d}}{\partial \boldsymbol{x}} \dot{\boldsymbol{x}}
\end{aligned}
$$

where $\boldsymbol{h}_{e}, \boldsymbol{h}_{d}$ are respectively the encoder and decoder neural network functions, while $\boldsymbol{x}, \tilde{\boldsymbol{q}}$ are respectively the obtained minimal coordinates and reconstructed full multibody coordinates.

The novelty is that the autoencoder training includes the multibody physics information [3]. In this way, the autoencoder does not only perform a dimensionality reduction of the original coordinates but can be used for model order reduction. The obtained reduced-order model consists of ordinary differential equations (with respect to the original differential algebraic equations) and can be used to perform dynamic simulations and easily coupled with standard estimation algorithms for stateestimation.

A typical application example is a mechanism with closed-loops, such as a car suspension, for which no analytical expression is available to reduce the full multibody model to minimal coordinates. The introduced physics-informed neural network allows to reduce the model and combine it with an augmented extended Kalman filtering scheme [4], in order to estimate difficult-to-measure inputs or parameters in the system.

In a first application, the model in combination with measured strut displacement or acceleration, allows to estimate the strut force, in addition to the body full coordinates in position, velocity and acceleration. The rationale is reported in the figure below.

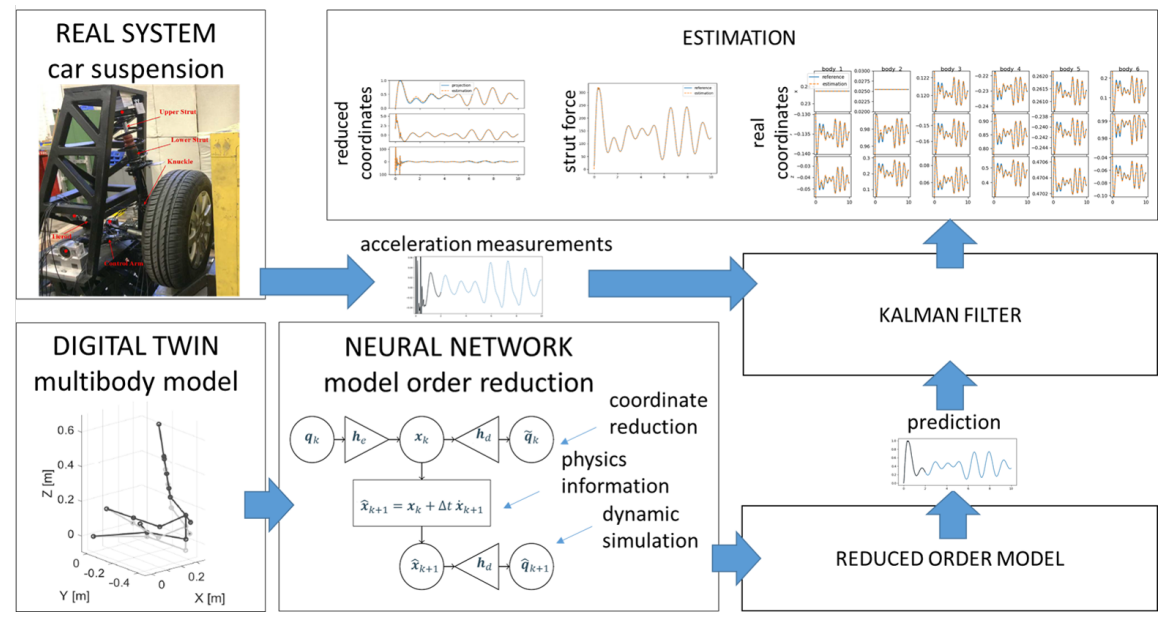

The Flanders Innovation \& Entrepreneurship Agency within the IMPROVED project and the AI impulse program of the Flemish Government are gratefully acknowledged for their support. 


\section{References}

[1] Javier Garcia de Jalón and Eduardo Bayo. Kinematic and dynamic simulation of multibody systems: the real-time challenge. Springer Science \& Business Media, 2012.

[2] Geoffrey E Hinton and Ruslan R Salakhutdinov. Reducing the dimensionality of data with neural networks. science, 313(5786):504$507,2006$.

[3] Andrea Angeli, Wim Desmet, and Frank Naets. Deep learning for model order reduction of multibody systems to minimal coordinates. Computer Methods in Applied Mechanics and Engineering, 373:113517, 2021.

[4] Dan Simon. Optimal state estimation: Kalman, H infinity, and nonlinear approaches. John Wiley \& Sons, 2006.

[5] Andrea Angeli, Frank Naets, and Wim Desmet. A machine learning approach for minimal coordinate multibody simulation. In Multibody Dynamics, pages 417-424. Springer, 2019.

[6] Andrea Angeli, Frank Naets, and Wim Desmet. Deep Learning of (Periodic) Minimal Coordinates for Multibody Simulations. volume Volume 2: 16th International Conference on Multibody Systems, Nonlinear Dynamics, and Control (MSNDC) of International Design Engineering Technical Conferences and Computers and Information in Engineering Conference, 082020. 